\title{
MODELOS DA SUPERFÍCIE POTENCIOMÉTRICA DO SISTEMA AQÜÍFERO CAIUÁ NO NOROESTE DO ESTADO DO PARANÁ: COMPARAÇÃO ENTRE KRIGAGEM ORDINÁRIA E KRIGAGEM COM TENDÊNCIA EXTERNA DO MODELO NUMÉRICO DO TERRENO
}

\author{
POTENCIOMETRIC MODELS OF CAIUÁ AQUIFER SYSTEM IN PARANÁ STATE NORTHWEST: \\ COMPARISION BETWEEN ORDINARY KRIGING AND CO-KRIGING WITH EXTERNAL TENDENCY \\ FROM NUMERIC TERRAIN MODEL.
}

\author{
Daniel Fabian Bettú ${ }^{1}$ e Francisco José Fonseca Ferreira ${ }^{2}$
}

Recebido em 22/06/2004, aceito em 02/06/2005

\begin{abstract}
Resumo Modelos hidrogeológicos comprovam a existência de relação morfológica entre as superfícies topográfica e potenciométrica. O objetivo deste trabalho foi quantificar esta relação a partir da interpolação de dados potenciométricos e de relevo. Através da interpolação das cotas do nível estático, por meio de krigagem ordinária e krigagem com tendência do modelo numérico de terreno (MNT), foram criados dois modelos da superfície potenciométrica de um aquífero livre (arenitos do Grupo Caiuá), na área compreendida entre as coordenadas $23^{\circ} 00^{\prime}$ e $24^{\circ} 00^{\prime}$ de latitude sul e 52 $30^{\prime}$ e $54^{\circ} 00^{\prime}$ de longitude oeste. Os dados utilizados foram fornecidos pela Companhia de Saneamento do Paraná (Sanepar) e pela Superintendência de Desenvolvimento de Recursos Hídricos e de Saneamento Ambiental (Suderhsa), referentes a 230 poços produtores. As técnicas empregadas para a interpolação envolveram a análise exploratória dos dados, a qual orientou a geração dos variogramas utilizados nas etapas subseqüientes. Após as interpolações foram feitas análises comparativas dos resultados obtidos. Esta comparação utilizou a técnica da validação cruzada, permitindo relacionar os dados medidos e calculados para as mesmas posições. Através da avaliação quantitativa e qualitativa dos resultados da validação cruzada, verificou-se que os melhores resultados foram obtidos pelo o método de krigagem com tendência do MNT, em relação ao método de krigagem ordinária. A baixa densidade de pontos de amostragem dificultou a realização das análises variográficas, e das krigagens, impedindo a contemplação de feições geomorfológicas importantes no controle da superfície potenciométrica. Por fim constatou-se que a superfície potenciométrica selecionada apresenta morfologia semelhante ao relevo da área.
\end{abstract}

Palavras-chave: Sistema Aqüífero Caiuá, geoestatística, potenciometria, hidrogeologia, modelo numérico de terreno.

\begin{abstract}
The hydrogeologic models provide evidence of morphological relation between topographic and potentiometric surfaces. The aim of this work was to quantify this relation from the interpolation of potentiometric and relief data. Through static level height interpolation, using ordinary kriging and kriging with trend from the terrane numeric model (TNM), were created two potentiometric surface models of a free-aquifer (Caiuá Group arenites), located in the region located in coordinates $23^{\circ} 00^{\prime}$ and $24^{\circ} 00^{\prime}$ south and $52^{\circ} 30^{\prime}$ and $54^{\circ} 00^{\prime}$ west. The data were supplied by Companhia de Saneamento do Paraná (Sanepar) and Superintendência de Desenvolvimento de Recursos Hídricos e de Saneamento Ambiental (Suderhsa), and refer to 230 flow wells. The techniques used for the interpolations involved a probing analysis of the data, which guided the construction of the variograms used in the following steps. After the interpolations, a comparative analysis of obtained results was made. This comparative analysis used the cross-validation technique, allowing us to associate the measured and calculated data for the same locations. Through qualitative and quantitative cross-validation results evaluation, it was concluded that the best results were obtained by kriging with trend from the TNM method, as compared to the ordinary kriging. The low density of sampling points made the variographic analysis and kriging more difficult, hindering the contemplation of geomorfological features important for the potentiometric surface control. Lastly, it was found that the selected potentiometric surface presents a similar morphology to the area landscape.
\end{abstract}

Keywords: Caiuá Aquifer System, geoestatistics, water table, hidrogeology, terrain numeric model.

\section{INTRODUÇÃO}

A região noroeste do estado do Paraná é caracterizada basicamente por rochas de composição arenosa, pertencentes ao Grupo Caiuá, Bacia Bauru (FERNANDES; COIMBRA, 1996), denotando porosidades e permeabilidades elevadas, facilitando assim a percolação e o armazenamento das águas subterrâneas do Sistema Aqüífero Caiuá, que na região é classificado por Rosa Filho (1982) como do tipo livre e semi-confinado. $\mathrm{Na}$ área-alvo deste estudo, localizada entre as coordenadas $23^{\circ} 00^{\prime} \mathrm{e}$ $24^{\circ} 00^{\prime}$ Sul e $52^{\circ} 30^{\prime}$ e $54^{\circ} 00^{\prime}$ Oeste (cerca de $17.000 \mathrm{~km}^{2}$ ), foram considerados 230 poços produtivos, os quais são utilizados para atividades agro-industriais e abastecimento domiciliar. Os dados de cota do nível estático foram cedidos pela Companhia de Saneamento do Paraná (SANEPAR, 2002) e pela Superintendência de Desenvolvimento de Recursos Hídricos e de Saneamento Ambiental (SUDERHSA, 2002), e constam dos seguintes parâmetros: coordenadas planas UTM, cota altimétrica da boca do poço, tipo de aqüífero e profundidade do nível estático.

\footnotetext{
TCurso de Pós-Graduação em Geologia Exploratória, UFPR, Bolsista CT-HIDRO/CNPq (daniel_bettu@yahoo.com.br)

${ }^{2}$ Laboratório de Pesquisas em Geofísica Aplicada-LPGA, DEGEOL-UFPR (francisco.ferreira@ufpr.br)
} 


\section{OBJETIVO}

Este trabalho pretende comparar o resultado de dois modelos digitais da superfície potenciométrica da porção sudeste do Grupo Caiuá no estado do Paraná gerados através da utilização de procedimentos de geoestatística. Os modelos potenciométricos foram construídos através da interpolação dos dados de nível estático dos poços por krigagem ordinária (KO) e krigagem com tendência externa (KTE) do MNT. As atividades envolveram a retirada da tendência regional dos valores da cota altimétrica do nível estático, em função de seu posicionamento e do modelo numérico do terreno (MNT) além do cálculo da correlação espacial entre os mesmos por meio da análise variográfica. As krigagens (KO e KTE) foram realizadas utilizando seus respectivos variogramas. Ao fim, através dos mapas de erros de krigagem, obtidos pela técnica da validação cruzada os resultados dos dois procedimentos foram comparados.

\section{MATERIAIS E MÉTODOS}

O primeiro passo da pesquisa foi a avaliação exploratória do banco de dados (SANEPAR, 2002; SUDERHSA, 2002) e a análise da existência de dependência espacial dos valores obtidos, através da geração de mapas de tendência através de regressões polinomiais. A interpolação dos dados foi realizada por $\mathrm{KO}$ e KTE, esta última utilizando a covariável MNT. Por fim os resultados obtidos foram comparados através da técnica da validação cruzada, que permitiu calcular os respectivos erros de interpolação, pela diferença entre os valores medidos e estimados para um determinado ponto.

Uma comparação visual entre os modelos gerados também foi possível através da geração de mapas de contorno das superfícies interpoladas.

Os métodos utilizados consistiram de uma série de tratamentos estatísticos e geoestatísticos, além de manipulações e cálculos simples em tabelas, e foram desenvolvidas em meio computacional utilizando basicamente os aplicativos $R^{\odot}$ 1.5.0 (R Development Core Team, 2005) - incluindo os pacotes MASS, geoR e methods, todos eles com código aberto, e Surfer ${ }^{\mathcal{Q}}$ 8 (Golden Software, 2002), ambos em ambiente Windows $^{\mathcal{\Theta}}$ (Microsoft).

Inicialmente se verificou a consistência dos dados originais, através da construção de histogramas e da avaliação de sua distribuição, utilizando os resultados da transformação BoxCox (BOX; COX, 1964), que forneceram uma estimativa da transformação logarítmica mais adequada para o tratamento dos dados, verificando assim o quanto as transformações logarítmicas conseguem aproximar a distribuição dos dados do nível estático a uma distribuição normal.

Tendo em vista que a distribuição dos dados mostrou uma tendência regional de decréscimo dos valores em direção ao noroeste da área (Figura 5) e que a densidade dos mesmos não refletia a variabilidade espacial da superfície potenciométrica (Figura 2), dois procedimentos foram adotados: (1) subtração da tendência do MNT (KTE) e por regressão polinomial (KO) e krigagem dos resíduos.

Foi efetuada a subtração da tendência espacial dos dados através de uma regressão polinomial de segunda ordem (polinômio de $2^{\circ}$ grau) para a krigagem ordinária; a krigagem com tendência externa do MNT foi realizada com a retirada da tendência do relevo. Em ambos os casos os variogramas foram construídos sobre os resíduos das tendências. Da mesma forma as krigagens foram realizadas sobre os valores dos resíduos, os quais foram somados posteriormente aos respectivos mapas de tendência, tanto para a KO quanto para a KTE.

As interpolações resultaram em dois grids que foram comparados a fim de determinar qual método mais preciso em suas predições, através da técnica de validação cruzada, conforme a equação:

$$
\mathrm{E}=\mathrm{I}-\mathrm{O}
$$

onde $\mathrm{E}$ é o erro, I é o valor interpolado e $\mathrm{O}$ é o valor observado, estes para um mesmo ponto.

Também foram comparados qualitativamente com o MNT os mapas de contorno das superfícies potenciométricas geradas.

\section{Análise exploratória}

Os dados estudados são provenientes de 230 poços produtores de água situados na região noroeste do estado do Paraná (Figura 1). A distribuição espacial destes poços pode ser visualizada na Figura 2.

As cotas dos poços variam de $231 \mathrm{~m}$ a 600 $\mathrm{m}$, com média em $363 \mathrm{~m}$ e desvio padrão de 65 $\mathrm{m}$, com uma tendência decrescente em direção à calha do rio Paraná. Os histogramas (figuras 3 e 4) mostram uma distribuição de freqüência aproximadamente simétrica para a altitude, com moda na classe $350-400 \mathrm{~m}$ e assimétrica para a cota do nível estático, com moda também entre $350-400 \mathrm{~m}$. 


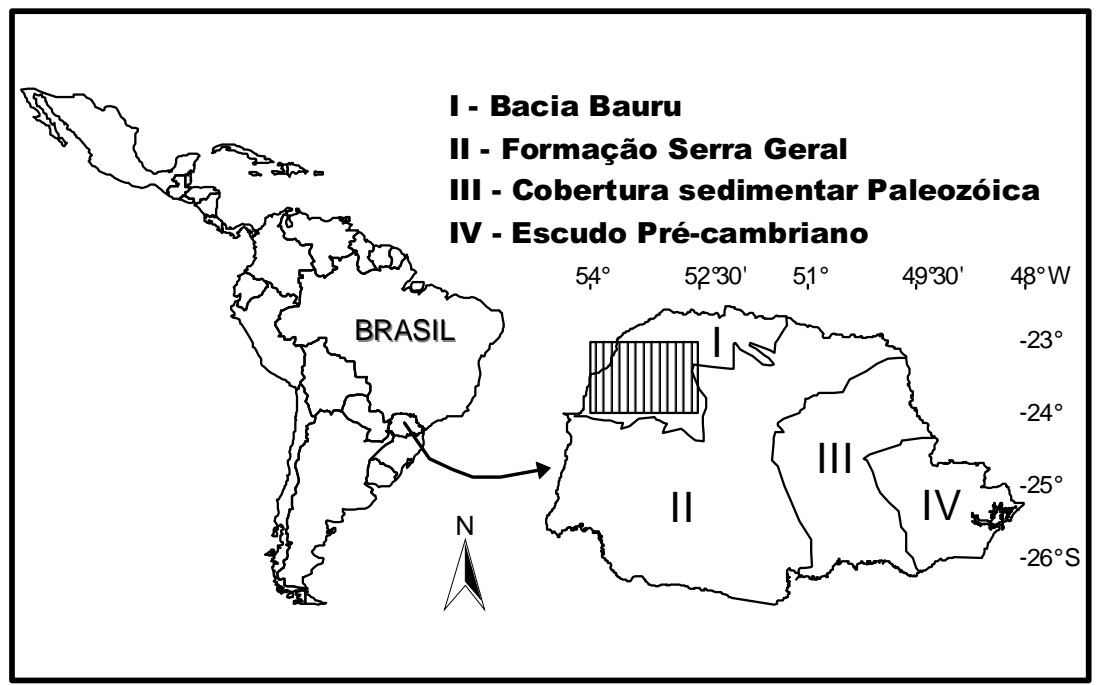

Figura 1 - Localização da área de estudo e contexto geológico.

Figure 1 - Study area location and geological context.

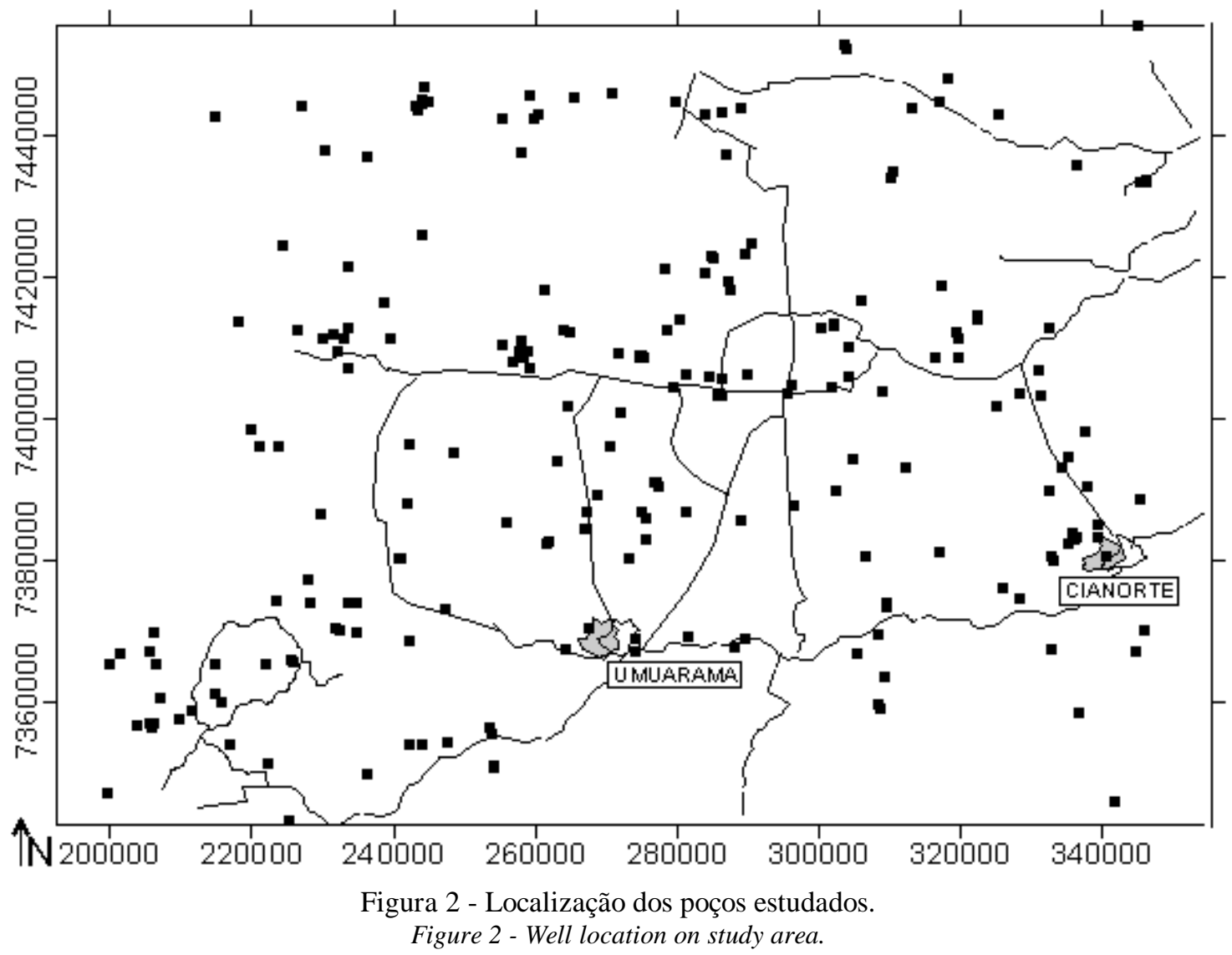


Modelos da superfície potenciométrica do Sistema Aqüífero Caiuá no noroeste do Estado do Paraná: comparação entre krigagem ordinária e krigagem com tendência externa do modelo numérico do terreno

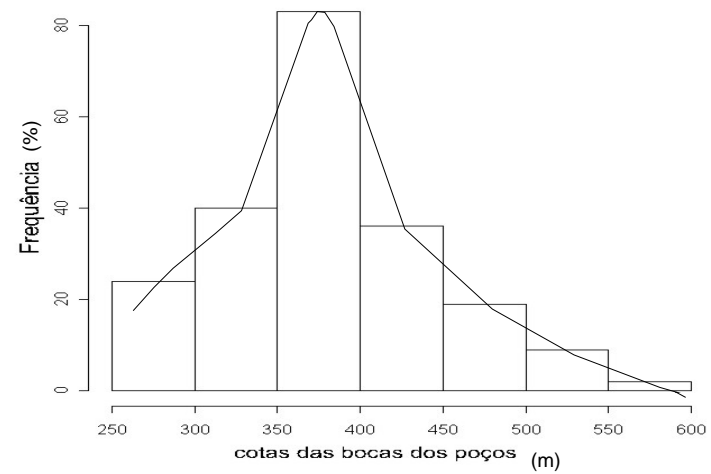

Figura 3 - Histograma das cotas dos poços estudados (em metros).

Figure 3 - Opening wells altitude histogram (meters).

\section{Análise de tendência espacial dos dados}

Os dados foram verificados quanto à existência de tendência espacial por regressões polinomiais de primeira, segunda e terceira ordem, onde se verificou que o polinômio de segunda ordem conseguiu gerar uma superfície

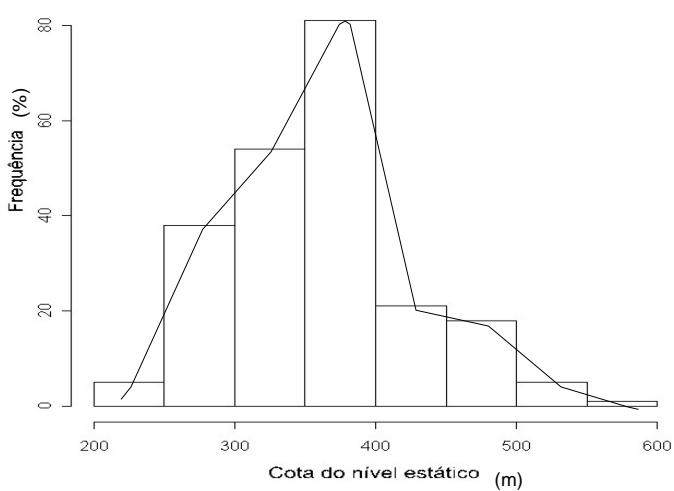

Figura 4 - Histograma das cotas do nível estático (em metros).

Figure 4 - Static water level altitude histogram (meters).

de tendência condizente com o esperado para os dados (Figura 5). O mapa da superfície de tendência mostra os valores mais elevados na porção sudeste, com tendência de decréscimo para noroeste, coincidindo com a direção do eixo do rio Paraná.

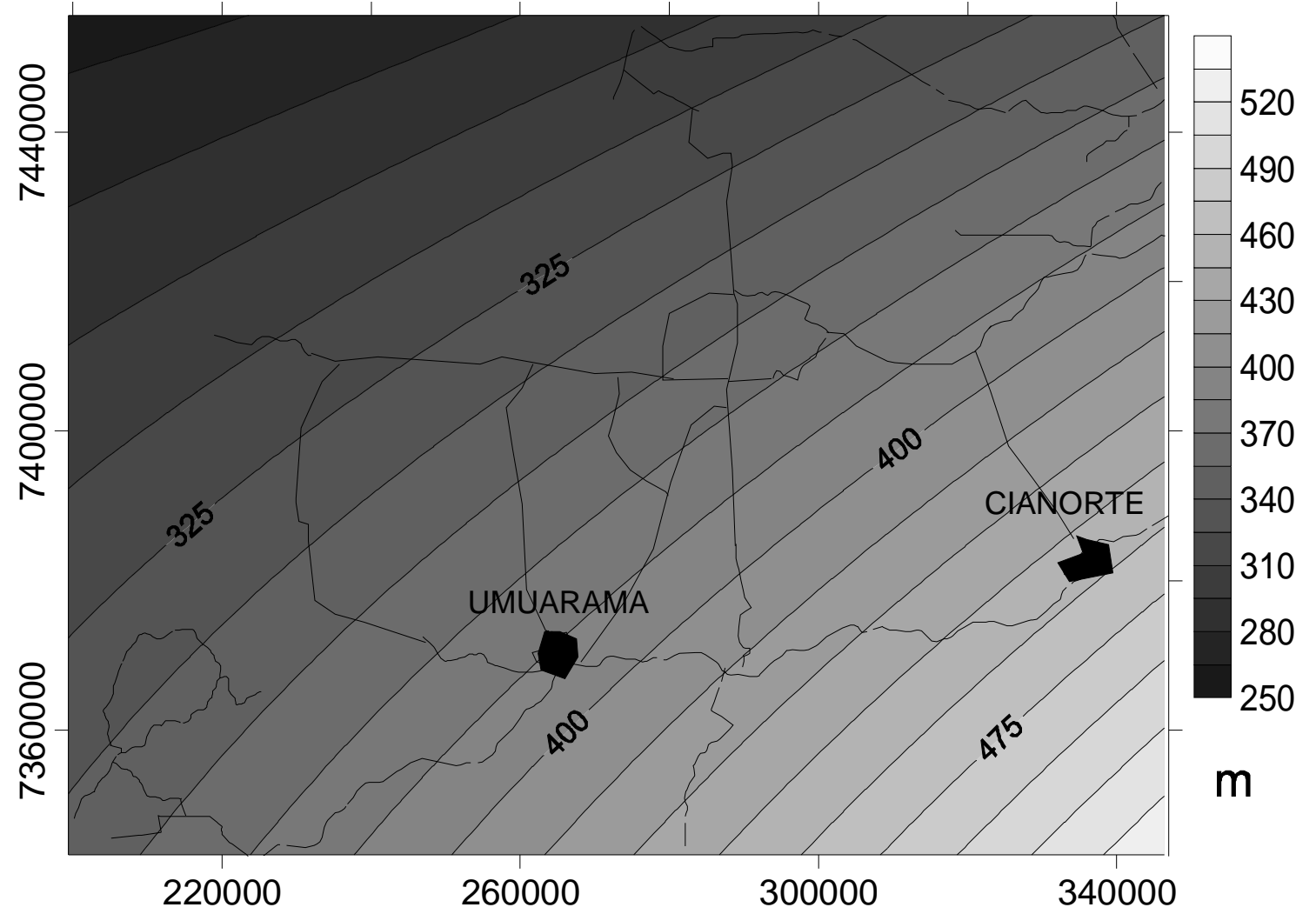

Figura 5 - Mapa de contorno da superfície de tendência de segunda ordem dos dados potenciométricos originais.

Figure 5 - Contour map of second order surface trend of potenciometric data. 


\section{Transformação Box-Cox}

A transformação Box-Cox (BOX; COX, 1964) avalia a eficiência das transformações logarítmicas em aproximar a distribuição dos

dados a uma curva normal. Foram testados os dados originais do nível estático, os resíduos das regressões de primeira e segunda ordem, além daqueles provenientes da subtração da superfíciede tendência externa do MNT (Figura $6)$.

Quando lambda fica localizado próximo ao valor zero é indicada uma transformação logarítmica na base neperiana para aproximar a distribuição dos dados a uma distribuição normal; quando o centro do intervalo de máxima confiança $(95 \%)$ se localiza próximo ao valor 1 , os dados originais podem ser trabalhados sem necessidade de transformação, pois já possuem uma distribuição adequada. A Figura 6A mostra a transformação Box-Cox aplicada aos dados originais, sem retirada de tendência. Nota-se que seria indicada uma transformação logarítmica natural para seu tratamento estatístico. Para os resíduos de primeira ordem (Figura 6B), a janela de confiabilidade de $95 \%$ situa-se entre 0 e 1 , porém com o valor 1 (sem transformação) muito próximo ao limite inferior de confiabilidade. Já para os resíduos de segunda ordem, lambda é muito próximo a (Figura 6C), justificando a sua utilização para a análise espacial sem a necessidade de transformações adicionais. A transformação Box-Cox aplicada aos dados dos resíduos da tendência externa do MNT (Figura 6D) mostra um quadro semelhante àquele dos resíduos de segunda ordem (Figura 6C), não requerendo transformações logarítmicas adicionais (lambda ideal próximo ao valor 1).

\section{Análise variográfica}

Sobre os resíduos de segunda ordem foi calculado um variograma direcional (Figura 7), utilizado na krigagem ordinária subseqüente. Tal variograma foi construído com uma distância máxima dos passos (lags) de $33.000 \mathrm{~m}$, largura de cada um deles de $2.200 \mathrm{~m}$, com base no seguinte estimador "clássico":

$$
\gamma=1 / 2 N \sum_{1}^{N}\left(Z_{x}-Z_{x+\Delta x}\right)^{2}
$$

onde $Z_{x}$ representa $o$ valor da variável no primeiro ponto; $\mathrm{x}$ é a posição do primeiro ponto; $\mathrm{x}+\Delta \mathrm{x}$ é é a posição do segundo ponto $(\Delta \mathrm{x}$ representa o valor do passo $-2.200 \mathrm{~m}$ ) e $\mathrm{N}$ indica o número total de pares. O variograma foi interpretado como possuindo um efeito pepita inicial muito reduzido, próximo ao valor 16 , ao qual foi ajustado um modelo exponencial, com patamar próximo de $1.810 \mathrm{~m}$ e alcance em torno de $6.000 \mathrm{~m}$. O ajuste foi realizado inicialmente de maneira visual, seguindo para um auto-ajuste disponível no aplicativo Surfer ${ }^{\odot} 8$ (Golden Software, 2002), o qual necessita da escolha de valores extremos para o efeito pepita, patamar e alcance do modelo. $\mathrm{O}$ auto-ajuste indicou também a presença de anisotropia no modelo exponencial, com maior continuidade na direção N65E, com razão entre o raio maior e o raio menor da elipse anisotrópica da ordem de duas vezes. A análise do variograma nas diferentes direções, com tolerâncias de $45^{\circ}$, cujos extremos são mostrados na Figura 7 (N-S - menor alcance e E-W - maior alcance), mostrou que os resultados dos ajustes ao modelo exponencial são satisfatórios para proceder a krigagem ordinária.

\section{Krigagem ordinária}

A partir dos variograma da Figura 7, foi realizada a interpolação por krigagem ordinária, utilizando as estimativas de dependência espacial definidas na etapa anterior, assim como a anisotropia do modelo variográfico.

As predições obedeceram a uma malha de 101 linhas por 114 colunas, totalizando 11.514 pontos estimados. A Figura 8 mostra o mapa de contorno resultante da krigagem ordinária, onde os valores mais acentuados situam-se na porção sudeste da área, decrescendo para norte-noroeste em direção aos rios Ivaí e Paraná.

A krigagem foi realizada sem a definição de uma elipse de busca de tamanho específico, utilizando assim todos os valores para a interpolação. Desta forma foi utilizada média móvel para o cálculo de pontos com afastamento maior que o alcance definido no variograma. 

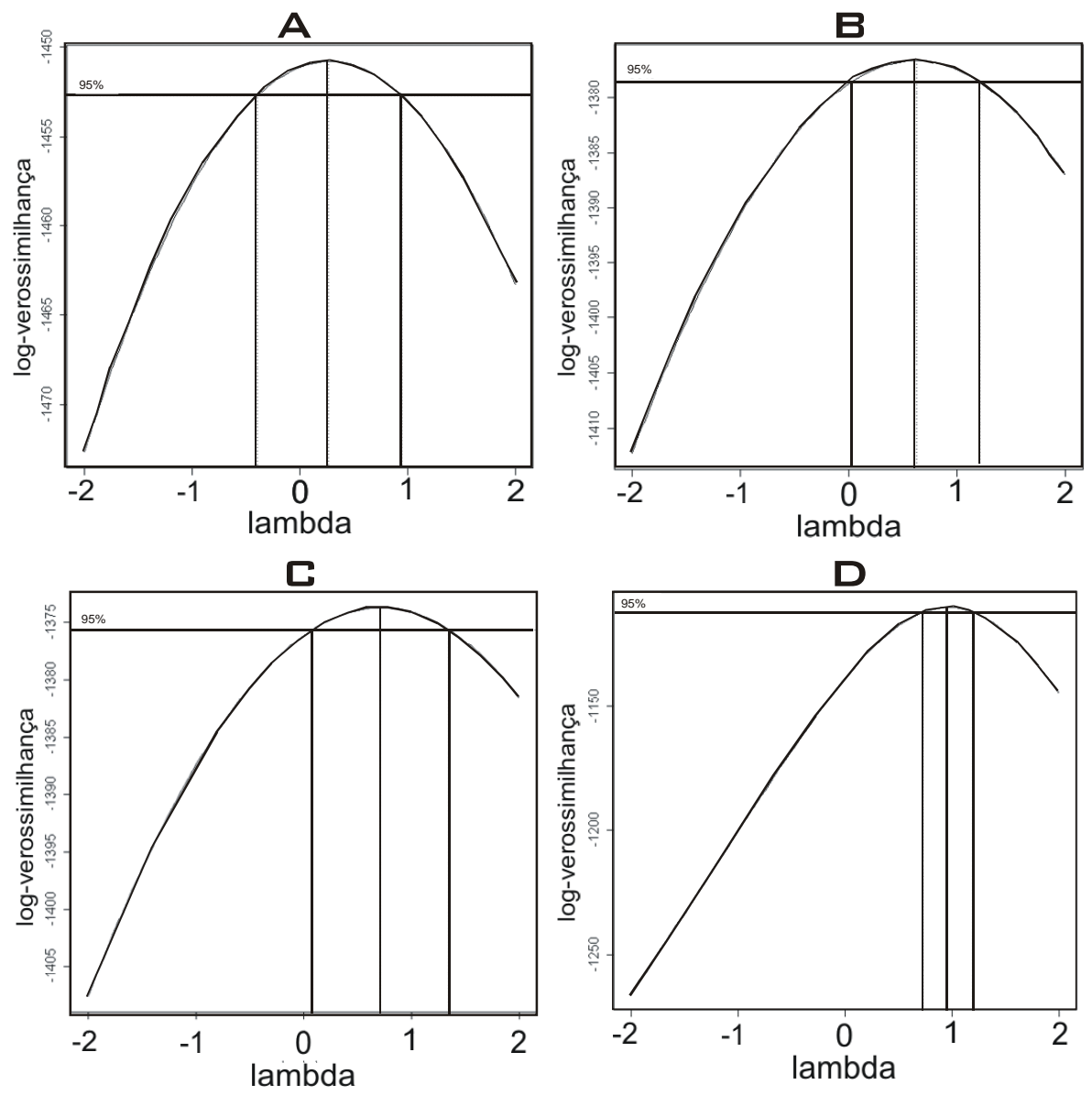

Figura 6 - Transformações Box-Cox aplicadas aos dados potenciométricos originais (A), aos resíduos da regressão polinomial de primeira ordem (B), de segunda ordem (C) e aos resíduos da tendência externa (D MNT). As linhas verticais marcam o intervalo de confiabilidade de $95 \%$.

Figure 6 - Box-Cox transformations applied to original potenciometric data (A), applied to residual from first order polynomial regression $(B)$, second order $(C)$ and to residuals of external tendency $(D)$. Vertical lines marks trust interval of $95 \%$.

Direção $90^{\circ}$ - Tolerância $45^{\circ}$

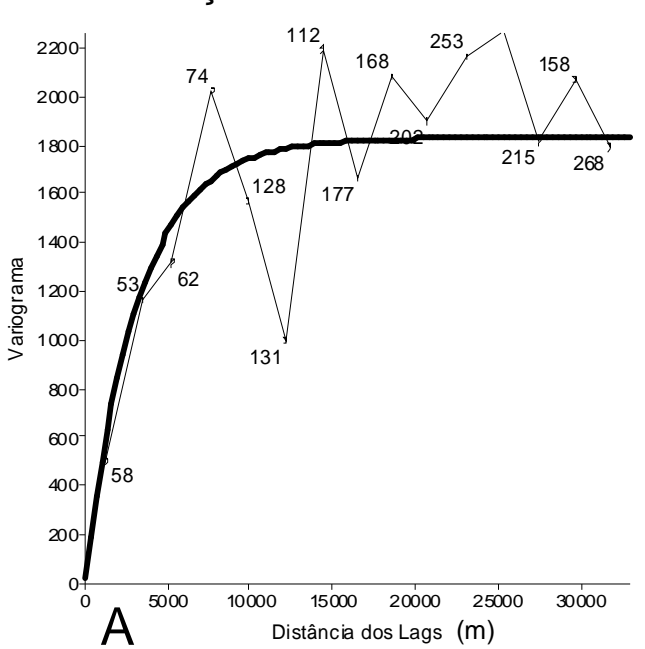

\section{Direção $0^{\circ}$ - Tolerância $45^{\circ}$}

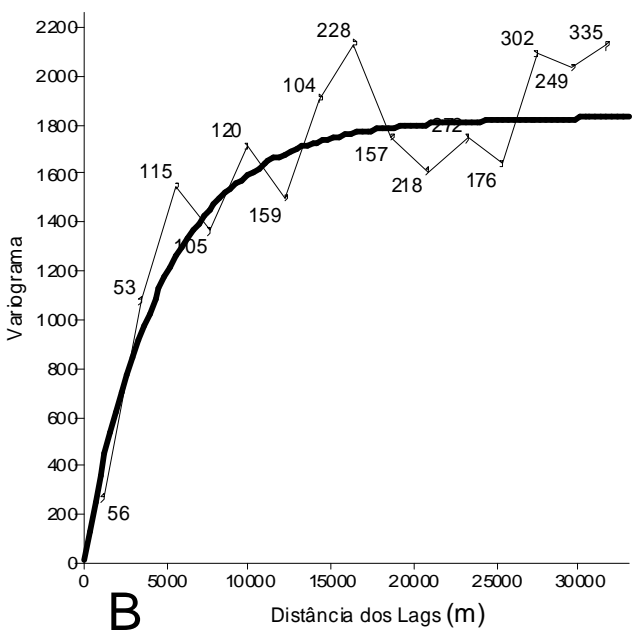

Figura 7 - Variograma dos resíduos da superfície de tendência de segunda ordem do nível estático, nas direçõe: $\mathrm{N}-\mathrm{S}(\mathrm{A})$ e E-W (B).

Figure 7 - Residual from second order trend variogram of static water level, to directions $N-S(A)$ and $E-W(B)$. 


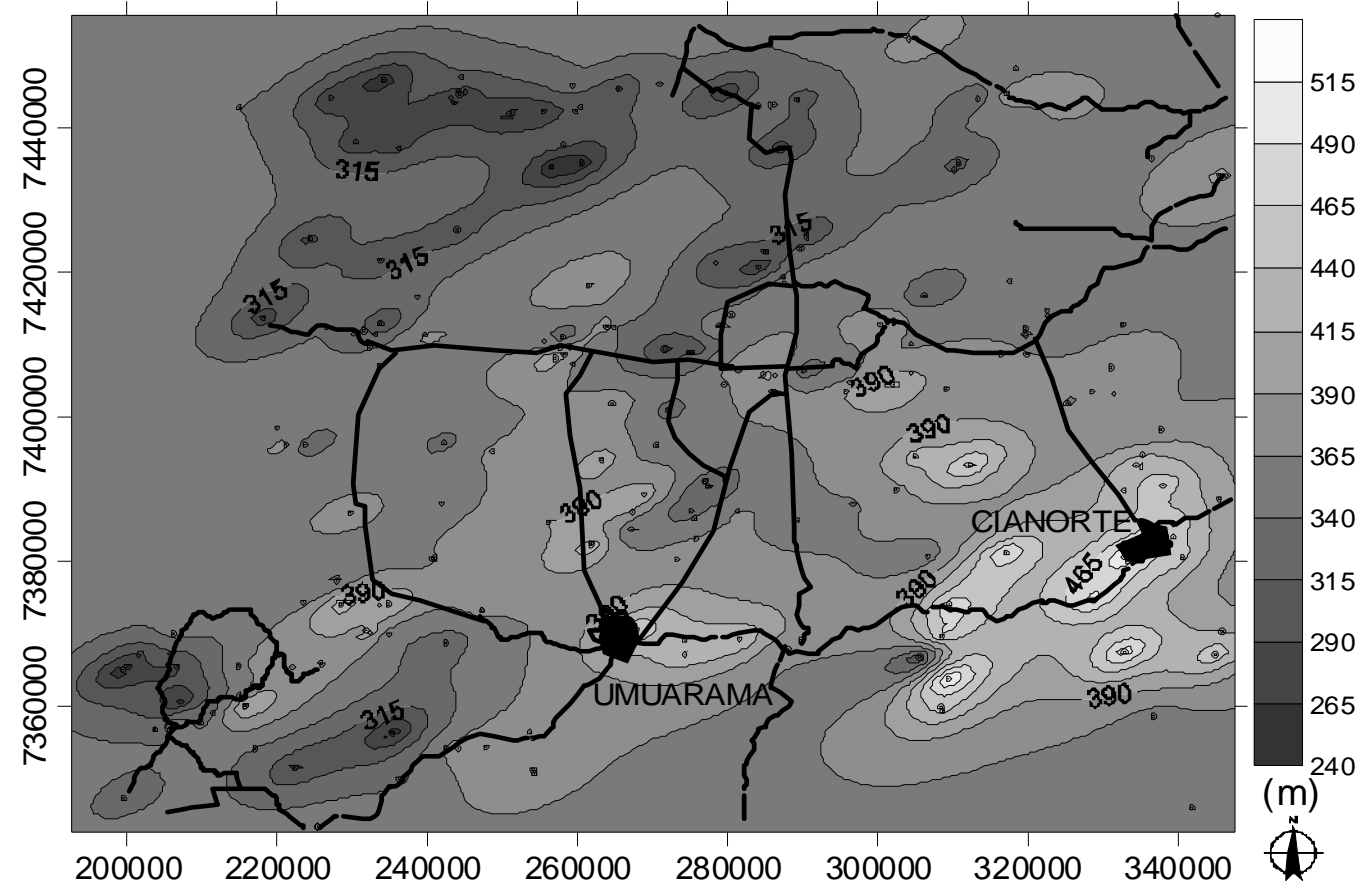

Figura 8 - Mapa de contorno da superfície potenciométrica gerado por krigagem ordinária dos resíduos de segunda ordem do nível estático.

Figure 8 - Contour map of potenciometric surface built by ordinary kriging of second order residuals of static water level.

\section{Validação cruzada}

Sobre os resultados da krigagem ordinária foi aplicado o procedimento de validação cruzada para o cálculo da diferença entre os valores calculados e medidos em campo, através da subtração do primeiro pelo segundo. A Tabela 1 mostra as estatísticas dos valores resultantes da subtração, e a distribuição das classes de valores é mostrada no histograma da Figura 9.

Tabela 1 - Estatísticas das diferenças entre os valores calculados pela krigagem ordinária e os valores medidos do nível estático.

Table 1 - Disparity statistics between calculate values by ordinary kriging and measured values of static water level.

\begin{tabular}{l|c}
\hline Valor mínimo & $-47,6 \mathrm{~m}$ \\
\hline Média & $27,595 \mathrm{~m}$ \\
\hline Valor máximo & $104,8 \mathrm{~m}$ \\
\hline Desvio padrão & $33,400 \mathrm{~m}$ \\
\hline
\end{tabular}

Análise da tendência externa - modelo numérico de terreno (MNT)

O nível estático é fortemente influenciado pelo relevo, uma vez que a recarga de aqüíferos do tipo livre ocorre através da infiltração direta a partir da superfície do terreno. Desta forma, uma outra maneira de visualizar o comportamento da superfície potenciométrica foi a inclusão, na análise, das cotas dos poços, na forma de covariável, uma vez que se verifica um boa correlação entre estes dados e os do nível estático (Figura 10), cujo coeficiente de correlação calculado por regressão linear foi de $\mathrm{R}=0,98$.

A krigagem com tendência externa exige o conhecimento do valor da covariável (altitude) nos pontos medidos e nos pontos onde serão realizadas as estimações, uma vez que a relação encontrada entre os dois valores em um determinado ponto medido é utilizada no cálculo de todos os demais pontos da malha interpolada. A construção do variograma foi realizada a partir dos resíduos do nível estático frente à tendência do MNT. A Figura 11 mostra o variograma orientado nas direções N-S, NE-SW, E-W e NW$\mathrm{SE}$, exibindo anisotropia direcional da variância de cerca de duas vezes entre as direções NE-SW (menor alcance - Figura 11B) e NW-SE (maior alcance - Figura 11 D). A anisotropia direcional foi contemplada na KTE. O ajuste do modelo variográfico exponencial (linha contínua no variograma da Figura 11) foi realizado automaticamente pelo aplicativo $R^{\odot}$ 1.5.0, com base em janelas de valores estimados previamente pelo usuário para o efeito pepita, alcance e patamar. 
Modelos da superfície potenciométrica do Sistema Aqüífero Caiuá no noroeste do Estado do Paraná: comparação entre krigagem ordinária e krigagem com tendência externa do modelo numérico do terreno

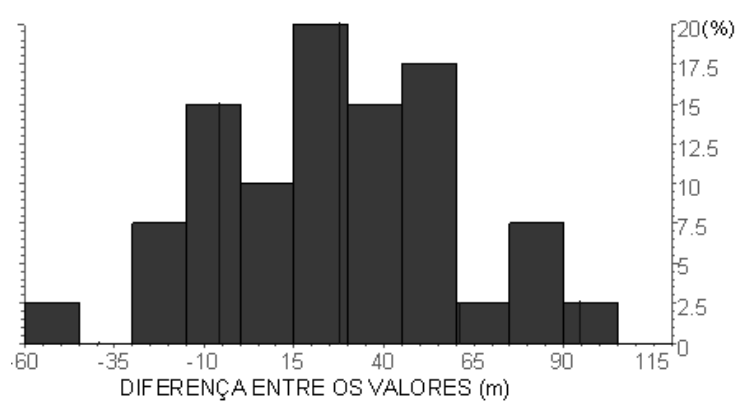

Figura 9 - Histograma de freqüência das diferenças entre os valores calculados pela krigagem ordinária e os valores do nível estático medidos (classes de 15 $\mathrm{m})$.

Figure 9 - Frequency histogram of disparity between calculate values by ordinary kriging and measured values of static water level (15 m class).

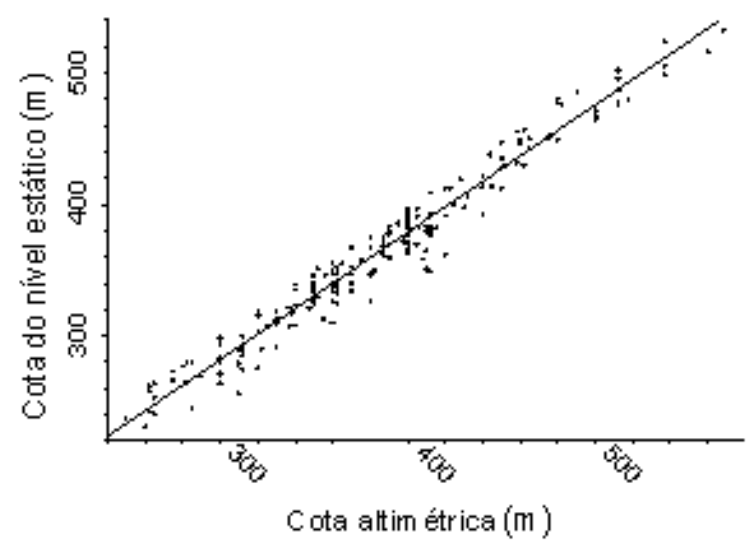

Figura 10 - Gráfico de correlação entre as cotas dos poços e do nível estático. Coeficiente de correlação $\mathrm{R}=0,98$.

Figure 10 - Correlogram between opening wells altitude and static water level altitude. Correlation coefficient $R=0.98$.
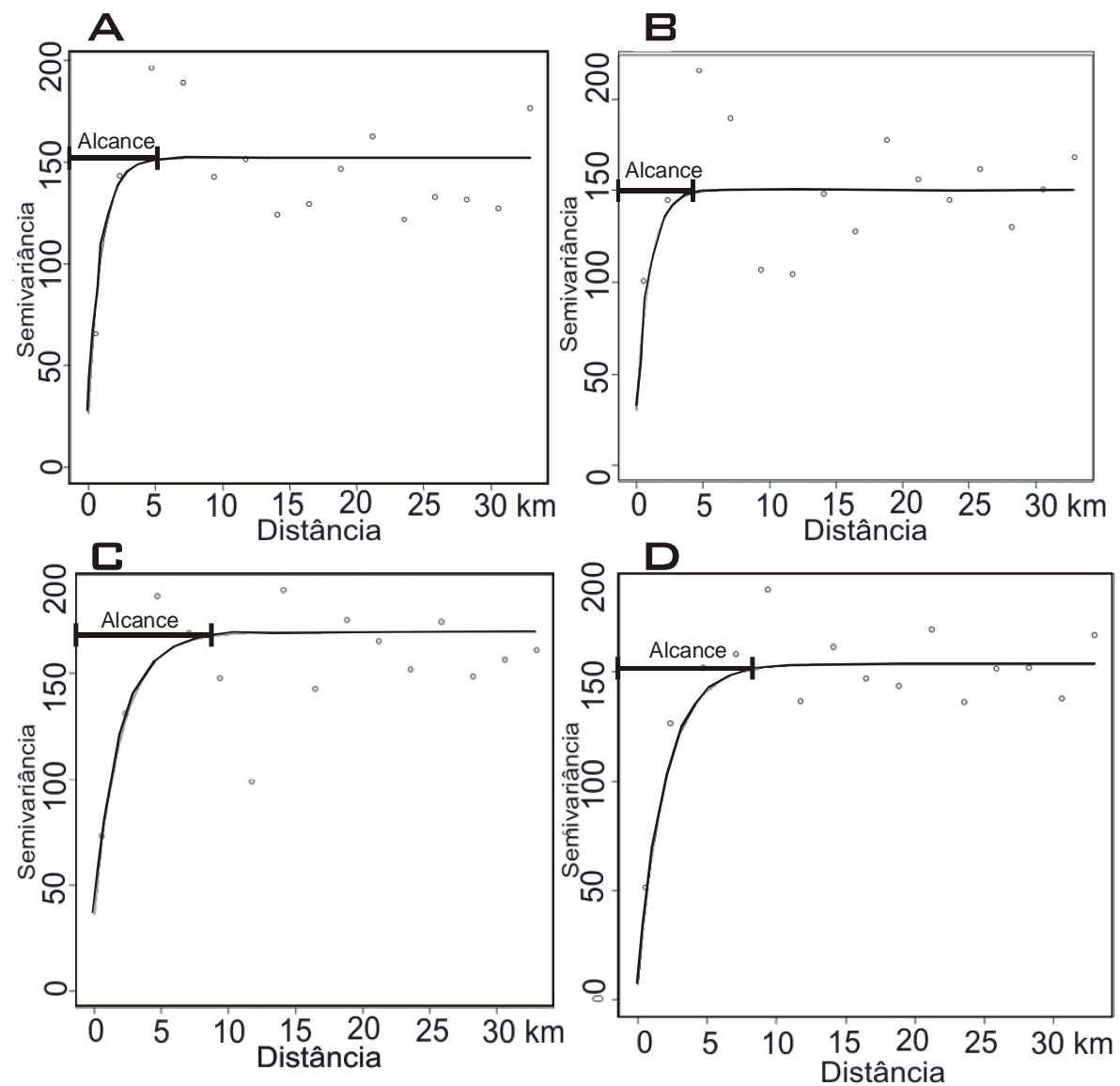

Figura 11 - Variograma dos resíduos da tendência externa (MNT) segundo as direções N-S (A), NE-SW (B), EW (C) e NW-SE (D). A linha contínua representa o modelo exponencial ajustado pelo aplicativo.

Figure 11 - Variogram of residuals from external tendency of Numeric Terrain Model to directions $N-S(A), N E-S W(B), E$ $W(C)$ and $N W-S E(D)$. Full line represent exponential model adjusted. 


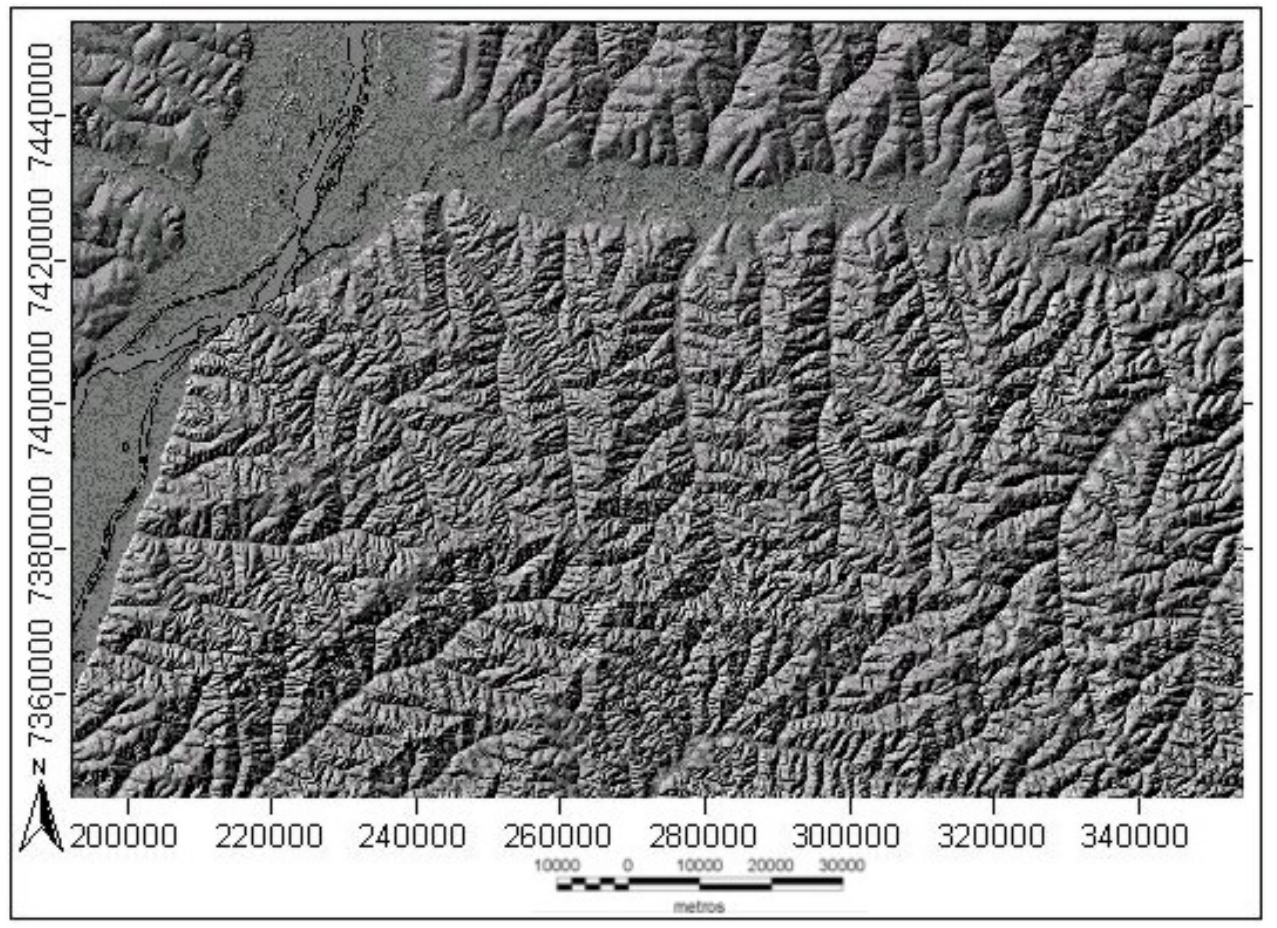

Figura 12 - Representação do modelo numérico do terreno (MNT) da área estudada. Figure 12 - Shaded relief of numeric terrain model.

\section{Krigagem com tendência externa}

Os dados originais do nível estático foram krigados fazendo uso do variograma anisotrópico construído sobre os resíduos da tendência externa do MNT. O MNT pode ser observado na Figura 12.
As predições foram realizadas seguindo uma malha de 51 linhas por 51 colunas, totalizando 2.601 pontos estimados e resultaram no mapa de contorno mostrado na Figura 13. É possível observar a semelhança entre o MNT (Figura 12) e o mapa de contorno resultante da krigagem com tendência externa (Figura 13).

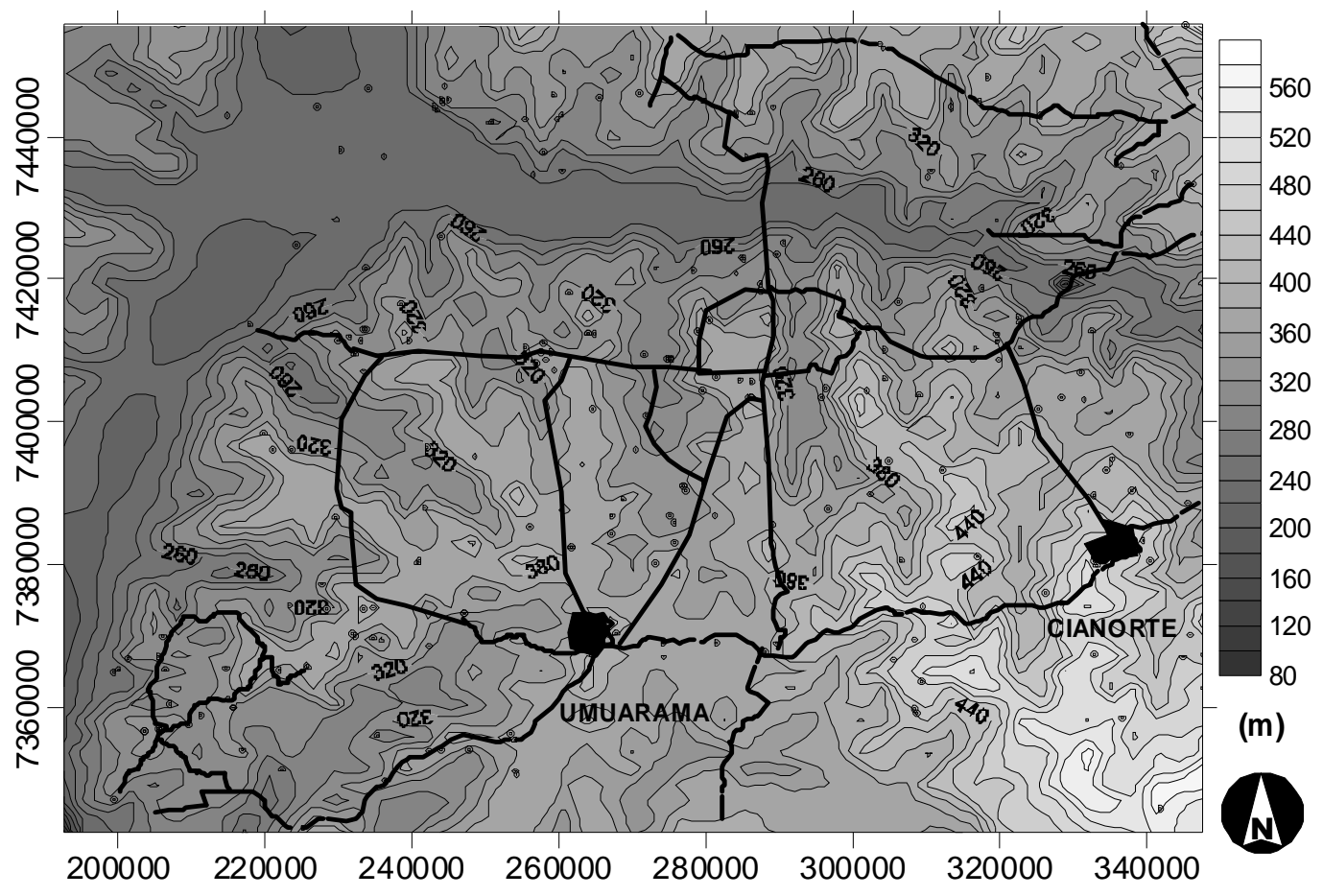

Figura 13 - Mapa de contorno da superfície potenciométrica, construído através de krigagem com tendência externa do MNT.

Figure 13 - Contour map of potenciometric surface, built by co-kriging with external tendency of numeric terrain model. 


\section{Validação cruzada}

Sobre o grid gerado pela krigagem com tendência externa, realizou-se o procedimento de validação cruzada. Como na análise da validação cruzada resultante da krigagem ordinária, foram analisadas as diferenças entre os valores calculados e medidos em campo, através da subtração do primeiro pelo segundo.

As estatísticas dos valores resultantes da subtração são exibidos na Tabela 2, enquanto a distribuição das classes de valores é mostrada no histograma da Figura 14.

Tabela 2 - Estatísticas das diferenças entre os valores calculados pela krigagem com tendência externa do MNT e os valores do nível estático medidos.

Table 2 - Disparity statistics between calculate values by co-kriging with external tendency of numeric terrain model and measured values of static water level.

\begin{tabular}{l|c}
\hline Valor mínimo & $-149,8 \mathrm{~m}$ \\
\hline Média & $1,025 \mathrm{~m}$ \\
\hline Valor máximo & $161,2 \mathrm{~m}$ \\
\hline Desvio padrão & $41,697 \mathrm{~m}$ \\
\hline
\end{tabular}

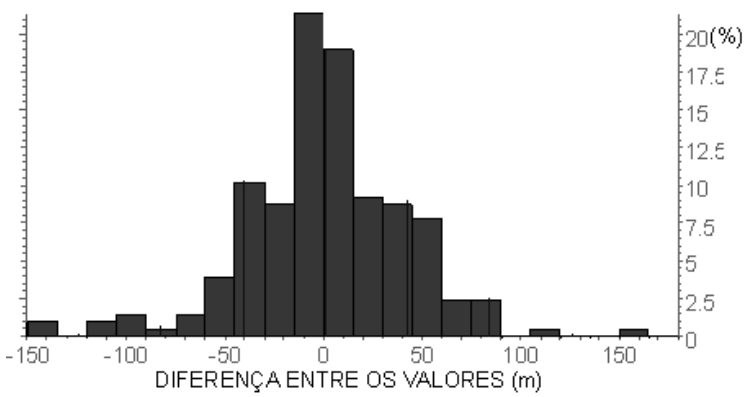

Figura 14 - Histograma de freqüência das diferenças entre os valores calculados pela krigagem com tendência externa do MNT e os valores do nível estático medidos (classes de $15 \mathrm{~m}$ ).

Figure 14 - Frequency histogram of disparity between calculated values by co-kriging and measured values of static water table (15m class).

\section{DISCUSSÃO DOS RESULTADOS CONCLUSÕES}

A relação entre o relevo e a superfície potenciométrica de aqüíferos do tipo livre devese a recarga a partir da superfície e a capilaridade dos solos, onde as duas superfícies tendem a apresentar morfologias semelhantes.

Com base nessa premissa foi realizada uma comparação entre os dois métodos de interpolação utilizados.

Foram comparadas as estatísticas dos resultados da validação cruzada, para os dois procedimentos de krigagem adotados (krigagem ordinária e krigagem com tendência externa do MNT), bem como de seus respectivos histogramas.

Embora a diferença entre os valores calculados e medidos para a krigagem ordinária tenha atingindo um range menor que a krigagem com tendência do MNT $(152,4 \mathrm{~m}$ para a primeira e $311,0 \mathrm{~m}$ para a segunda), a segunda obteve maior êxito quanto a média destes valores $(1,02$ $\mathrm{m})$ enquanto a primeira apresentou um viés de $27,5 \mathrm{~m}$ para o erro médio.

A análise dos histogramas mostrados nas figuras 9 e 14, respectivamente representando as diferenças de valores medidos e calculados para a krigagem ordinária e com tendência externa, mostra que primeira apresenta classe modal entre 15 e $30 \mathrm{~m}$ e a segunda entre -15 e $0 \mathrm{~m}$.

A comparação visual entre os mapas de contorno gerados a partir dos dois procedimentos de interpolação (figuras 8 e 13), mostrou melhores resultados no caso da krigagem com tendência externa, exibindo maior semelhança em sua morfologia com o MNT, conforme sugerem os modelos hidrogeológicos de aquíferos do tipo livre. Isto é explicado pelo fato da krigagem com tendência externa do MNT permitir um maior controle na interpolação em locais distantes dos pontos amostrados. A KTE estima o valor do nível estático para um determinado ponto afastado das amostras com maior precisão uma vez que utiliza a altitude deste ponto para proceder a estimação, o que não acontece na krigagem ordinária, a qual tende a aproximar o valor estimado à média dos valores medidos, a medida que aumenta a distância dos pontos com informação.

Embora estatisticamente a krigagem ordinária tenha apresentado valores ligeiramente melhores que a krigagem com tendência do MNT (range de valores da diferença entre os valores medidos e calculados - tabelas 1 e 2), a análise da morfologia dos dois modelos potenciométricos gerados (figuras 8 e 13) revela que regionalmente a krigagem com tendência do MNT apresentou os melhores resultados, corroborado pelos erros médios da validação cruzada (tabelas 1 e 2). $\mathrm{O}$ fato de haver uma diferença entre os resultados da avaliação quantitativa e qualitativa é explicado pela grande distância entre os pontos amostrados, uma vez que a krigagem ordinária tende a aproximar a estimação de valores ao valor médio da variável com o incremento da distância em relação às amostras.

Devido ao número de amostras contempladas pela análise que decorreu a 
existência de grande distância entre os pontos amostrados não foi possível a contemplação numérica de fatores adicionais que certamente interferem no comportamento da superfície potenciométrica, como distância entre o interflúvio e o fundo do vale e a diferença de altitude entre a área de recarga do aqüífero e os poços. Desta forma os variogramas referentes as duas krigagens procedidas utilizaram distância de passos (lags) muito superiores às distâncias ideais para análises variográficas mais eficazes.

Pode-se concluir que a interpolação dos dados por krigagem com tendência externa do MNT apresentou resultados satisfatórios no delineamento da superfície potenciométrica em toda a extensão da área de estudo, com maior fidelidade ao relevo que a interpolação por krigagem ordinária. Resultados semelhantes podem ser esperados para outros aquíferos do tipo livre, principalmente quando a densidade de amostras permita contemplar com maior eficácia os fatores geomorfológicos que controlam o comportamento hidrogeológico da região.

Da mesma maneira como foi utilizado o MNT como tendência externa na krigagem da superfície potenciométrica, outras covariáveis podem ser incluídas no cálculo, necessitando apenas que seja equacionada a sua relação com a variável a ser interpolada. A krigagem com tendência externa mostrou-se uma importante ferramenta de interpolação espacial, não somente para de dados hidrogeológicos, mostrando-se de grande utilidade principalmente quando a distribuição espacial dos dados não sejam satisfatórias e exista uma covariável que permita facilitar sua estimação.

\section{REFERÊNCIAS}

BOX, G. E. P.; COX, D. R. An Analysis of Transformations, Journal of the Royal Statistical Society. Oxford, p. 211-252, 1964.

FERNANDES, L.A.;COIMBRA, A.M. A Bacia Bauru (Cretáceo Superior, Brasil). Anais da Academia Brasileira de Ciências, Rio de Janeiro, n. 68, vol 2, p. 195-205, 1996.

GOLDEN SOFTWARE. Surfer Version 8.00 Surface Mapping System. Golden Software, Inc., 2002.

R DEVELOPMENT CORE TEAM. R: A Language and Environment for Statistical Computing. $\mathrm{R}$ Foundation for Statistical Computing. Vienna, Austria, 2005.
ROSA FILHO, E. F. Uma Análise sobre a Produtividade de Poços Tubulares no Aqüífero Caiuá - Região Noroeste do Estado do Paraná. Recife, 1982. Dissertação (Mestrado em Geologia). Centro de Tecnologia, Universidade Federal de Pernambuco, 394p.

SANEPAR (Companhia de Saneamento do Paraná). 2002. Banco de Dados Hidrogeológico, Curitiba, CDROM.

SUDERHSA (Superintendência de Desenvolvimento de Recursos Hídricos e de Saneamento Ambiental). 2002. Banco de dados hidrogeológicos, Curitiba, CDROM. 
Modelos da superfície potenciométrica do Sistema Aqüífero Caiuá no noroeste do Estado doPparaná: comparação entre krigagem ordinária e krigagem com tendência externa do modelo numérico do terreno 